\title{
Uso de medicamentos entre gestantes de um município no sul de Minas Gerais, Brasil
}

\author{
Use of medications among pregnant women in a city in the \\ southern region of the Minas Gerais, Brazil
}

Recebido em: 02/06/2017

Aceito em: $\quad 02 / 11 / 2017$
Vinícius Miranda BORGES ${ }^{1}$; Fernanda MOURA ${ }^{1}$; Cláudio Daniel CERDEIRA ${ }^{2}$; Gérsika Bitencourt Santos BARROS ${ }^{1}$ ${ }^{1}$ Faculdade de Medicina, Universidade José do Rosário Vellano (UNIFENAS). Rodovia Km 0, Bairro Trevo, MG 179, CEP 37130000. Alfenas, MG, Brasil. ${ }^{2}$ Departamento de Bioquímica, Instituto de Ciências Biomédicas, Universidade Federal de Alfenas. Rua Gabriel Monteiro da Silva, 700, CEP 37130-000. Alfenas, MG, Brasil.E-mail: daniel.cerdeira.84@gmail.com

\section{ABSTRACT}

This cross-sectional study identified, through home interviews, the pharmacotherapeutic profile of pregnant women attended at the Unified Health System (SUS) during the prenatal care. The drugs were classified according to the Food and Drug Administration (FDA) pregnancy risk categories. From the 104 respondents, $94.2 \%$ reported to use at least one drug during pregnancy, and were identified 53 different drugs, totalizing 316 . The frequency of drugs from category $\mathrm{A}(n=115,36.4 \%)$ was closely related to $\mathrm{B}(n=121,38.3 \%)$ and 3 times higher than $\mathrm{C}(n=36,11.4 \%)$ and 4 than $\mathrm{D}(n=29,9.2 \%) .22 .1 \%$ (CI $(95 \%) 14.4-29.8)$ of the respondents used drugs from category $\mathrm{D}$ and $1.9 \%$ from X. There was a significant association between gestational age and drug use, so that $61.7 \%$ of the drugs were consumed in the third trimester of gestation, and $56.7 \%$ of the respondents consumed drugs at this period. The most commonly consumed therapeutic classes were vitamins/supplements/antianemics (35.33\% from the 316 ; consumed by $72.97 \%$ of the interviewed), painkillers $(14.6 \% ; 44 \%)$, and antimicrobials $(10.1 \% ; 19.2 \%)$. The most frequently consumed drugs were folic acid (17.72\% CI(95\%) 13.67 - 22.39), ferrous sulfate (15.82\% CI(95\%) 11.98 - 20.32\%), and paracetamol $(12.97 \% \mathrm{CI}(95 \%) 9.47$ - 17.19). From the 104 respondents, $22 \%$ related smoking before pregnancy and $12.5 \%$ did not interrupt the habit during pregnancy, whereas 21 pregnant women related alcohol consumption before pregnancy and, 4 them, did interrupt the consumption during the gestational period. Therefore, this study reported a great number of prescribed drugs to pregnant women, from all FDA pregnancy risk categories, including an outstanding use of drugs with a high teratogenic potential ( $\mathrm{D}$ and $\mathrm{X}$ categories), and a considerable proportion of pregnant women using more than one drug. The control and supervision during the use of drugs at the prenatal care must be taken into account, since the use of certain drugs during gestational period may lead to teratogenic damage and/or complications that cause abort and impair the pregnant woman's health.

Keywords: Pregnant women; drugs; risk groups

\section{RESUMO}

Neste estudo transversal, por meio de entrevistas domiciliares, foi mostrado o perfil farmacoterapêutico de gestantes atendidas pelo Sistema Único de Saúde (SUS) durante o pré-natal, sendo que os medicamentos utilizados foram classificados segundo critérios de risco do Food and Drug Administration (FDA). Entre as 104 gestantes entrevistadas, 94,2\% utilizavam medicamento e foram identificadas 53 especialidades farmacêuticas no total de 316 medicamentos (média de 3 medicamentos por gestante). A frequência de medicamentos do grupo A $(n=115,36,4 \%)$ foi próxima ao $\mathrm{B}(n=121,38,3 \%), 3$ vezes maior que o $\mathrm{C}(n=36,11,4 \%)$ e 4 que o $\mathrm{D}(n=29,9,2 \%) .22,1 \%(\mathrm{IC}(95 \%) 14,4-29,8)$ das entrevistadas utilizavam medicações da classe D e 1,9\% da X. Houve uma positiva e significativa correlação entre a 
idade gestacional e uso de medicação, com $60,1 \%$ dos medicamentos sendo consumidos no terceiro trimestre, e 56,7\% das gestantes utilizando-os neste período. As classes terapêuticas mais consumidas foram: vitaminas/suplementos minerais/preparações anti-anêmicas (35.33\% dos 316 medicamentos; 72,97\% das entrevistadas), analgésicos (14,6\%; $44 \%$ das gestantes) e agentes anti-infecciosos de uso sistêmico $(10,1 \% ; 19,2 \%$ das gestantes), e os medicamentos foram: ácido fólico (17,72\% IC(95\%) 13,67 - 22,39), sulfato ferroso (15,82\% IC(95\%) 11,98 - 20,32) e paracetamol (12,97\% IC(95\%) 9,47 - 17,19). 22\% das 104 entrevistadas relataram o hábito de tabagismo antes da gestação e, 12,5\% continuaram com o hábito durante o período gestacional, enquanto que 21 gestantes faziam uso de bebidas alcoólicas antes da gestação e, entre estas, 17 interromperam o consumo durante a gestação. Portanto, é reportado nesse estudo um grande número de medicamentos prescritos para gestantes, constante nas 5 classes de risco do FDA, incluindo uma grande quantidade de medicamentos das classes de alto risco teratogênico (D e X), e uma considerável proporção de gestantes utilizando mais de um medicamento. O controle e supervisão do uso de medicamentos devem ser levados em consideração durante os cuidados no pré-natal, visto que o consumo de certos medicamentos durante a gestação pode acarretar em danos teratogênicos e/ou complicações que causam aborto e comprometem a saúde da gestante.

Palavras-chave: Gestantes; fármacos; grupos de riscos.

\section{INTRODUÇÃO}

O período vital do ser humano é constituído por diversas etapas. Para as mulheres, uma destas etapas pode representar a oportunidade de abrigar uma nova vida, processo conhecido como gravidez. Este processo, juntamente com o parto e o puerpério, é reconhecido como fisiológico, mas traz mudanças comportamentais acompanhado de mudanças físicas, psicológicas e uma diferenciada vivência para a mulher (1-4).

Durante a gestação, complicações podem ocorrer, nas chamadas "gestações de alto risco", um termo primeiramente descrito nos anos 60 , como referência à falta de condições que o ambiente materno poderia oferecer ao feto, ou doenças ligadas ao próprio zigoto (5). Neste mesmo período, estima-se que cerca de $10 \mathrm{mil}$ crianças recém-nascidas foram diagnosticadas com focomelia devido ao uso de talidomida durante a gestação. A partir de então, a utilização de medicamentos durante a gestação passou a ser objeto de preocupação e alvo de diferentes estudos, visando o uso seguro bem como a prevenção quanto ao uso de fármacos nocivos para a gestante e/ou o feto (6).

A saúde reprodutiva da mulher no Brasil ainda é considerada precária. Com intuito de modificar essa realidade, o Ministério da Saúde instituiu, em junho de 2000, o Programa de Humanização do Pré-natal e Neonatal (PHPN) (7). Este programa visava reduzir as altas taxas de morbi-mortalidade materna perinatal e neonatal, ampliar o acesso ao pré-natal, estabelecer critérios para qualificar as consultas e, também, promover o vínculo entre a assistência ambulatorial na atenção primária e o parto (7).

Assim, no Brasil, o acompanhamento pré-natal deve seguir as recomendações para a atenção básica, caso não tenham sido identificados riscos, propiciando atenção primária em unidades básicas de saúde (UBSs) inseridas na Estratégia Saúde da Família (ESF) e ambulatórios do Sistema Único de Saúde (SUS). O indício de qualquer risco resulta em um exame/avaliação e seguimentos adicionais para a atenção especializada, e, se necessário, a referência da atenção básica para um serviço de nível mais complexo. A assistência pré-natal envolve objetivos específicos e bem delimitados, garantindo a evolução fisiológica da gravidez, preparando a gestante para o futuro parto, puerpério e lactação e, além disso, identificando situações de risco, portanto, propiciando uma intervenção precisa e precoce de complicações que podem ocorrer durante a gestação (8-11).

Neste contexto, quando é necessário o uso de fármacos por gestantes, precauções devem ser tomadas visando a promoção da saúde da mãe e prevenção de possíveis danos teratogênicos ou complicações que possam causar aborto. Segundo a Organização Mundial de Saúde (OMS), o uso racional de medicamentos ocorre quando pacientes recebem medicamentos apropriados para suas condições clínicas, em doses adequadas às suas necessidades individuais, por um período adequado, e ao menor custo para si e para a comunidade $(12,13)$. O medicamento é visto como uma resposta imediata e simples para condições que requerem ações individuais, e muitas vezes sociais para sua completa resolução (12). Durante a gravidez, a exposição a um determinado fármaco envolve não apenas a mãe, como também o feto, que é muito mais sensível aos seus efeitos e toxicidade, podendo sofrer graves problemas, até mesmo aqueles irreversíveis (13).

O uso de medicamentos durante a gestação constitui um problema de grande magnitude para a saúde pública. Grande parte dos fármacos administrados durante a gestação pode atravessar a barreira placentária, portanto expondo o embrião em desenvolvimento a seus 
efeitos. Um fármaco quando produz, direta ou indiretamente, alteração na morfologia ou fisiologia do feto ou da criança após o nascimento, pode ser considerado teratogênico (14).

Quando alguns específicos grupos de medicamentos são usados no primeiro trimestre da gestação, essas alterações, principalmente má formação congênita, possuem maiores chances de ocorrer (13). Portanto, de acordo com a agência governamental Norte Americana, Food and Drug Administration (FDA), os fármacos são classificados quanto ao seu potencial risco teratogênico $(14,15)$. Entretanto, o número de gestantes que fazem uso de medicamentos sobre os quais não há informação sobre segurança na gravidez pode chegar a $79 \%$ (14). Neste sentido, a utilização de medicamentos durante a gravidez deve ser vista com cautela e, estar sob criteriosa avaliação do risco/benefício, devido às implicações sobre a saúde do feto (15).

Diante do exposto, embora o uso de medicamentos durante a gestação seja um tema de extrema relevância para a saúde pública, raros estudos, conduzidos em apenas algumas cidades brasileiras, têm sido publicados (6, 13-17). Portanto, levantamentos que identifiquem o uso de medicamentos pelas gestantes, em diferentes localidades do país, se tornam de extrema importância. Neste estudo foi estimada a frequência de mulheres grávidas usando medicação, as frequências de distribuição dos medicamentos usados (classificados de acordo com o FDA), e a potencial associação do uso de medicamentos e variáveis de interesse.

\section{MÉTODOS}

Aspectos éticos. Esse estudo foi realizado de acordo com os padrões éticos constantes na Resolução 466/12 do Conselho Nacional de Saúde (CNS), e foi previamente aprovado pelo comitê de ética em pesquisa envolvendo humanos (Protocolo de aprovação: CAAE 32009514.4.0000.5143) da Universidade José do Rosário Vellano (UNIFENAS-Alfenas). O protocolo do estudo foi desenhado para proteger as entrevistadas de possíveis riscos. As gestantes selecionadas foram convidadas a participar deste estudo e esclarecidas a respeito dos propósitos do mesmo, e um termo de consentimento livre e esclarecido (TCLE) foi assinado por cada participante.

Amostragem. Nesse estudo foram incluídas todas as gestantes atendidas em 14 ESF e 4 ambulatórios de Alfenas, MG, Brasil, entre setembro de 2014 e janeiro de 2015. Portanto, a população amostral foi composta por gestantes maiores de 18 anos, incluindo gestantes de baixo ou alto risco, cadastradas no SUS e atendidas pela rede pública de saúde (ESF e ambulatórios do município), totalizando 104 gestantes $(n=104)$.

Desenho do estudo. Esse é um inquérito de caráter observacional e transversal, realizado por meio de entrevistas domiciliares com gestantes que responderam a um questionário estruturado, entre setembro de 2014 e janeiro de 2015. Os entrevistadores foram previamente calibrados (estatística kappa $>0,8$ ) e no momento das entrevistas não possuíam conhecimento sobre o risco da gestante.

Quadro 1. Variáveis* coletadas entre gestantes de Alfenas, MG, Brasil.

\begin{tabular}{|c|c|}
\hline Dados & Variável \\
\hline \multirow{2}{*}{ 1. Demográficos } & - Idade \\
\hline & - Etnia \\
\hline \multirow{3}{*}{ 2. Socio-econômicos } & - Escolaridade \\
\hline & - Ocupação \\
\hline & - Renda familiar \\
\hline \multirow{4}{*}{ 3. Condições de saúde } & -História obstétrica (Idade gestacional) \\
\hline & - Tabagismo \\
\hline & - Uso de álcool \\
\hline & - Uso de substâncias ilícitas \\
\hline \multirow{3}{*}{ 4. Uso de fármacos e outros agentes terapêuticos } & - Medicamentos (estratificados por classes FDA)** \\
\hline & - Uso de chás \\
\hline & - Outras substâncias utilizadas durante a gestação (excluindo vacinas) \\
\hline
\end{tabular}

*Antes/durante a gestação; **Durante a gestação; Variáveis: dependentes (uso de medicamentos, classe farmacológica,- se prescrito ou não) e independentes (idade, escolaridade, ocupação, renda familiar, tabagismo, uso de álcool e trimestre da gestação em que foi usado)

Os grupos farmacológicos dos medicamentos foram classificados de acordo com o sistema Anatomical Therapeutic Chemical (ATC) (OMS, 2013). Todas as
104 gestantes cadastradas nas unidades aceitaram previamente a participar do estudo, e nenhuma gestante abordada se recusou a responder questões do questio- 
nário e/ou completar o estudo. Todos os dados coletados foram registrados em questionários individuais. Ressalta-se a presença do viés de memória das entrevistadas. O período recordatório para as questões sobre o uso de medicamentos foi considerado durante a gravidez (medicamentos usados durante a gestação), sendo que as entrevistadas responderam à seguinte pergunta: "A senhora poderia me informar todos os medicamentos que utiliza/utilizou durante a gestação?". Em relação à idade gestacional, foi considerado primeiro trimestre até a $13^{\mathrm{a}}$ semana de gestação; segundo trimestre da $14^{\mathrm{a}}$ à $27^{\mathrm{a}}$ semana; e terceiro trimestre da $28^{\mathrm{a}}$ à $41^{\mathrm{a}}$ semana gestacional.

Análise dos dados. Os dados foram apresentados como valores absolutos e/ou percentagens e/ou médias. Para analisar possíveis relações casuais entre as variáveis coletadas, o uso de medicamentos (variável principal) foi correlacionado com outras variáveis provenientes dos dados sócio-demográficos e clínicos/farmacoterapêuticos (variáveis secundárias), como apresentado no Quadro 1. Para tal finalidade, o teste exato de Fisher ou Qui-Quadrado $\left(\chi_{2}\right)$ com o $p$-valor $(\boldsymbol{\alpha}=\mathbf{0 , 0 5})$ foi obtido por simulação de Monte Carlo, usando o software R, verificando possível dependência ou independência entre as variáveis, duas a duas. IC $(95 \%)$, para verificar quais medicamentos foram mais prevalentes e para quantificar o número de gestantes que utilizavam classes de risco foi obtido no software Sisvar. Foi também utilizado o IC exato para proporção utilizando a distribuição de $\mathrm{F}$.

Quadro 2. Classificação de risco dos fármacos*, de acordo com Food and Drug Administration.

\begin{tabular}{|c|c|}
\hline Classe/Categoria de risco FDA & Descrição da classe e de potenciais riscos \\
\hline A & $\begin{array}{l}\text { Estudos controlados realizados não demonstraram riscos para o feto durante o primeiro trimestre } \\
\text { de gravidez e não existem evidências de riscos em trimestres posteriores, sendo improvável a } \\
\text { possibilidade de teratogênese }\end{array}$ \\
\hline B & $\begin{array}{l}\text { Estudos em animais não demonstraram risco teratogênico, enquanto não se dispõem de estudos } \\
\text { controlados na gravidez humana; ou estudos em animais têm demonstrado efeitos teratogênicos } \\
\text { que não foram confirmados em grávidas humanas durante o primeiro trimestre da gestação, e não } \\
\text { existem evidências de riscos em trimestres posteriores. }\end{array}$ \\
\hline C & $\begin{array}{l}\text { Estudos em animais têm demonstrado efeitos teratogênicos sobre o feto e não existem estudos } \\
\text { em mulheres; ou não existem estudos disponíveis em mulheres, tampouco em animais. São } \\
\text { medicamentos que só devem ser administrados se o benefício esperado para a mãe justificar o } \\
\text { risco potencial para o feto. }\end{array}$ \\
\hline D & $\begin{array}{l}\text { Existem claras evidências de risco teratogênico, mas os benefícios acarretados pelo uso do } \\
\text { medicamento podem torná-lo aceitável. }\end{array}$ \\
\hline $\mathrm{x}$ & $\begin{array}{l}\text { Os estudos em animais ou em humanos demonstraram evidentes riscos de teratogênese, o que } \\
\text { claramente supera o possível benefício em gestantes. Os medicamentos dessa categoria estão } \\
\text { contraindicados em mulheres que estão ou poder ficar grávidas. }\end{array}$ \\
\hline
\end{tabular}

*Law e cols. (2010) (18)

\section{RESULTADOS E DISCUSSÃO}

Neste estudo foram entrevistadas 104 gestantes $(n=$ 104) atendidas pelo SUS no município de Alfenas, MG, Brasil. A média de idade das entrevistadas foi $27( \pm 7)$ anos, e a faixa etária mais frequente foi a de 25-34 anos. Quanto à etnia, a maioria das entrevistadas declarou ser leucoderma (45,2\%). Estes dados são apresentados em detalhes na Tabela 1 .

Quanto ao nível de escolaridade (Tabela 1), 100\% das gestantes entrevistadas eram alfabetizadas, sendo que $45,2 \%$ completaram o ensino médio e $36,6 \%$ completaram ao menos o fundamental. Foi encontrado um índice menor de gestantes que completaram ou que estavam cursando o ensino superior (apenas 8,7\% tinham superior completo), evidenciando talvez um padrão social brasileiro de que pessoas com escolaridade maior procuram menos os serviços do SUS, sendo que a outra justificativa poderia ser a taxa de natalidade menor entre pessoas que já atingiram maior nível educacional. A Tabela 1 também mostra uma estratificação relativa ao nível educacional das gestantes e a respectiva relação com o uso de medicamentos constantes nas classes de risco do FDA.

Em relação à ocupação e renda das entrevistadas (Tabela 1), foram considerados os serviços executados no momento da entrevista e também aquelas gestantes que saíram apenas momentaneamente por conta da gestação. A média de pessoas por residência foi de aproximadamente três. Com relação ao consumo de medi- 
camentos, Guerra e cols. (2008) observaram que ocorre um maior uso de medicamentos quanto maior a escolaridade e a renda familiar da mulher (14), ao contrário de outros estudos que relataram uma maior frequência de uso de medicamentos entre gestantes com menor escolaridade e maior número de filhos. Não houve associação significativa entre ocupação ou renda e o uso de medicamentos (Tabela 1).

Neste estudo foi observado que $94,2 \%(n=98)$ das entrevistadas utilizavam algum tipo de medicamento (Tabela 2), desde o início da gestação até a data em que foram entrevistadas, e a média do uso de medicamentos foi três por gestante sendo que algumas gestantes chegaram a utilizar medicamentos constantes em todas as classes de risco do FDA (Quadro 2) durante esse período (mais de 4 medicamentos). Um total de 53 especialidades farmacêuticas foi declarado pelas gestantes (Tabela 3), totalizando 316 medicamentos (Tabela 2). Cabe ressaltar que, alguns medicamentos contêm mais de um princípio ativo em suas formulações, o que os torna classificáveis em diferentes classes de risco do FDA (como mostrado na Tabela 2).

Tabela 1. Classificação socio-econômica e demográfica e condições de saúde entre as 104 entrevistadas, em Alfenas, MG, Brasil.

\begin{tabular}{|c|c|c|}
\hline Faixa etária & $\mathrm{N}^{0}$ entrevistadas & $\begin{array}{c}\% \text { entrevistadas } \\
(n=104)\end{array}$ \\
\hline Até 19 anos & 14 & 13,5 \\
\hline $19-24$ & 25 & 24 \\
\hline $25-34$ & 47 & 45,2 \\
\hline 35 ou mais que 35 anos & 18 & 17,3 \\
\hline 15-43 (Total) & 104 & 100 \\
\hline Média Idade (em anos, $n=104$ ) & 27 & \\
\hline $\mathrm{DP}$ & 7 & \\
\hline IC(95\%) & $\pm 1,37(25,63-28,37)$ & \\
\hline Etnia & $\mathrm{N}^{0}$ entrevistadas & $\begin{array}{c}\% \text { entrevistadas } \\
(n=104\end{array}$ \\
\hline Leucodermas & 47 & 45,2 \\
\hline Feodermas & 43 & 41,3 \\
\hline Melanodermas & 10 & 9,6 \\
\hline Xantodermas & 1 & 0,96 \\
\hline Não souberam informar & 3 & 2,9 \\
\hline Hábito & $\mathbf{N}^{0}$ entrevistadas & $\begin{array}{l}\% \text { entrevistadas } \\
(n=104)\end{array}$ \\
\hline \multicolumn{3}{|l|}{ Histórico de Tabagismo } \\
\hline Antes da gestação & 23 & 22 \\
\hline Continuou durante a gestação & 13 & 12,9 \\
\hline \multicolumn{3}{|l|}{ Uso de bebidas alcoólicas } \\
\hline Antes da gestação & 21 & 20 \\
\hline Continuou durante a gestação & 4 & 3,8 \\
\hline \multicolumn{3}{|l|}{ Tabagismo e Uso de bebidas alcoólicas } \\
\hline Antes da gestação & 11 & 10,58 \\
\hline Continuou durante a gestação & 1 & 0,97 \\
\hline \multicolumn{3}{|l|}{ Uso de substâncias ilícitas } \\
\hline Antes da gestação & 2 & 1,9 \\
\hline Continuou durante a gestação & 0 & 0 \\
\hline
\end{tabular}




\begin{tabular}{|c|c|c|c|c|c|c|c|}
\hline \multicolumn{8}{|c|}{ Escolaridade } \\
\hline & Fundamental & $\begin{array}{l}\text { Fundamental } \\
\text { incompleto }\end{array}$ & $\begin{array}{l}\text { Médio } \\
\text { completo }\end{array}$ & $\begin{array}{c}\text { Médio } \\
\text { incompleto }\end{array}$ & Superior & $\begin{array}{l}\text { Superior } \\
\text { incompleto }\end{array}$ & $\begin{array}{l}\text { Técnico } \\
\text { completo }\end{array}$ \\
\hline Classe FDA & \multicolumn{7}{|c|}{${ }^{*} \mathrm{~N}^{\circ}$ de gestantes } \\
\hline$A(75)^{* *}$ & 26 & 1 & 35 & 5 & 6 & 1 & 1 \\
\hline B (64) & 20 & 1 & 31 & 3 & 7 & 2 & 0 \\
\hline C (30) & 7 & 0 & 15 & 1 & 5 & 2 & 0 \\
\hline D (23) & 4 & 0 & 14 & 1 & 4 & 0 & 0 \\
\hline$x(2)$ & 0 & 0 & 1 & 0 & 1 & 0 & 0 \\
\hline
\end{tabular}

\begin{tabular}{|c|c|c|c|c|c|}
\hline Ocupação & $\begin{array}{c}\text { Empregada } \\
\text { com carteira } \\
\text { assinada }\end{array}$ & $\begin{array}{c}\text { Empregada } \\
\text { sem carteira } \\
\text { assinada }\end{array}$ & Desempregada & Estudante & Total \\
\hline$\%$ de gestantes & 24,04 & 19,23 & 50 & 6,73 & 100 \\
\hline
\end{tabular}

\begin{tabular}{|c|c|c|c|c|c|c|}
$\begin{array}{c}\text { Renda } \\
\text { Familiar*** }\end{array}$ & $\begin{array}{c}<1 \text { salário } \\
\text { minimo }\end{array}$ & $\begin{array}{c}\text { Até } 3 \text { salários } \\
\text { minimos }\end{array}$ & $\begin{array}{c}\text { Entre } 3 \text { e } \\
5 \text { salários } \\
\text { minimos }\end{array}$ & $\begin{array}{c}>5 \text { salários } \\
\text { minimos }\end{array}$ & $\begin{array}{c}\text { Não souberam } \\
\text { informar }\end{array}$ & \begin{tabular}{c} 
Total \\
\hline$\%$ de gestantes
\end{tabular} \\
\hline 5,88 & 75,85 & 13,46 & 2,88 & 1,92 & 100 \\
\hline
\end{tabular}

\begin{tabular}{|c|c|c|c|c|}
\hline \multicolumn{3}{|c|}{ Variáveis correlacionadas } & $p$-valor & $\begin{array}{l}\text { Relação causal } \\
\text { entre as variáveis }\end{array}$ \\
\hline \multicolumn{2}{|c|}{ Principal } & \multicolumn{3}{|c|}{ Secundária } \\
\hline \multirow{6}{*}{ 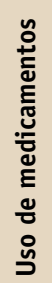 } & versus & Escolaridade & 0,7951\# & Independência \\
\hline & versus & Ocupação & $0,5547 \#$ & Independência \\
\hline & versus & Renda & $0,6522 \#$ & Independência \\
\hline & versus & Consumo de álcool & $0,6552 \#$ & Independência \\
\hline & versus & Hábito de fumar & $0,4358 \#$ & Independência \\
\hline & versus & Idade gestacional & $0,0111 \ddagger$ & Dependência \\
\hline
\end{tabular}

*Uma mesma gestante pode ter consumido mais de uma das classes de risco; ** Entre parênteses, o número absoluto de gestantes que usaram a respectiva classe; *** Soma dos salários de todos os membros do grupo familiar (salário mínimo em $2014=\mathrm{R} \$ 724,00$ ); $\neq$ Altamente significativo, de acordo com o teste exato de Fisher ou ${ }_{2} ;$ \#Não significativo com $\boldsymbol{\alpha}=0,05$

Proporções semelhantes foram encontradas em um grande levantamento envolvendo seis cidades brasileiras no início dos anos 2000 - Porto Alegre (1.110 gestantes), São Paulo (1.236), Rio de Janeiro (557), Salvador (985), Fortaleza (1.173), e Manaus (503) - no qual Mengue e cols. (2001), encontraram $4.614(83,8 \%)$ das 5.564 gestantes avaliadas entre a $21^{\mathrm{a}} \mathrm{e}$ a $28^{\mathrm{a}}$ semanas de gravidez sob uso de medicação, totalizando 9.556 medicamentos (16).

Em um estudo conduzido por Geib e cols. (2007), envolvendo 2.262 mulheres, entrevistadas na cidade de Passo Fundo, Rio Grande do Sul, Brasil, o consumo de medicamentos foi realizado por $80 \%$ das gestantes num total de 1.631 especialidades farmacêuticas (17). Em um estudo envolvendo 610 gestantes atendidas pelo SUS em uma das 13 unidades de saúde, na cidade de Natal,
Rio Grande do Norte, Guerra e cols. (2008) relataram o uso de pelo menos um fármaco na gravidez por $86,6 \%$ das entrevistadas (14). Também, Melo e cols. (2009), ao avaliarem 205 gestantes do município de Bandeirantes, Paraná, Brasil, observaram que $83,4 \%$ das entrevistadas usavam algum tipo de medicamento (6). Em outros países estes valores podem ser menores devido a protocolos durante a gestação e/ou as diferentes prevalências de doenças e agravos de saúde entre as gestantes (18).

Nesse estudo nenhuma gestante entrevistada afirmou estar sob automedicação, de modo que todos os medicamentos foram prescritos ou orientados por profissionais da área de saúde (médicos, enfermeiros e farmacêuticos). Por outro lado, Guerra e cols. (2008) mostraram que $12,2 \%$ das entrevistadas praticavam automedicação em Natal, Rio Grande do Norte (14). 
A maioria das entrevistadas $(69,2 \%)$ relatou não fazer uso de chás ou agentes terapêuticos tópicos, ao passo que os que foram relatados podem ser vistos na Tabela 4. De relevância clínica, alguns destes produtos relatados apresentam princípios ativos que são classificados quanto aos seus riscos pelo FDA.

Tabela 2. Relação dos 316 medicamentos, por classe de risco de acordo com o FDA, pesquisados entre 104 gestantes entrevistadas em Alfenas, MG, Brasil.

\begin{tabular}{|c|c|c|}
\hline \multirow{2}{*}{ Classificação FDA * } & \multicolumn{2}{|c|}{ Número de gestantes } \\
\cline { 2 - 3 } & N (\%) & IC $(95 \%)$ \\
\hline Usou Medicamento & $98(94,2)$ & $89,7-98,7$ \\
\hline A & $75(72,1)$ & $64,3-79,7$ \\
\hline B & $64(61,6)$ & $53,9-69,3$ \\
\hline C & $30(28,9)$ & $21,2-36,6$ \\
\hline D & $23(22,1)$ & $14,4-29,8$ \\
\hline X & $2(1,9)$ & $0-9,6$ \\
\hline SC & $11(10,6)$ & $2,9-18,3$ \\
\hline Não utilizou & $6(5,8)$ & $0-13,5$ \\
\hline Total de Medicamentos por Classe
\end{tabular}

\begin{tabular}{|c|c|c|}
\hline Classe & N $(\%)$ & IC(95\%) \\
\hline A & $115(36,4)$ & $29,76-43,47$ \\
\hline B & $121(38,3)$ & $31,8-45,28$ \\
\hline C & $36(11,4)$ & $7-16,92$ \\
\hline D & $29(9,2)$ & $5,79-14,02$ \\
\hline X & $2(0,63)$ & $0-3,1$ \\
\hline A+A+C*** & $1(0,32)$ & $0-1,5$ \\
\hline C+C+B+B**** & $1(0,32)$ & $0-1,5$ \\
\hline SC & $11(3,5)$ & $0-7,1$ \\
\hline Total & $316(100)$ & ------- \\
\hline
\end{tabular}

$\mathrm{SC}=$ sem classificação; *Alguns medicamentos continham mais de um principio ativo, portanto, classificados em mais de uma classe; ${ }^{* *} \mathrm{Me}$ dicamentos contendo mais que um princípio ativo de apenas uma das classes de risco foi contado apenas como $1 ; * * *$ Presença no medicamento de dois diferentes princípios ativos constantes na classe $\mathrm{A}$ e um na $C ; * * *$ Presença no medicamento de dois diferentes princípios ativos constantes na classe B e dois diferentes da C

Apesar do fato de que a aplicação tópica desses agentes terapêuticos pode diminuir a absorção dos princípios ativos e, consequentemente, minimizar possíveis danos à gestante e/ou ao feto, cautela é necessária quanto ao uso, principalmente no primeiro trimestre da gestação.

Tabela 3. Distribuição das 53 diferentes especialidades farmacêuticas com suas respectivas classes de risco FDA encontradas entre os 316 medicamentos utilizados por 104 gestantes de Alfenas, MG.

\begin{tabular}{|c|c|c|}
\hline Classe FDA & $\mathbf{N}^{0}$ & Fărmacos/principios ativos representantes \\
\hline A & 6 & $\begin{array}{l}\text { cloridrato de piperidolato + hesperidina + ácido ascórbico*; sulfato ferroso; ácido fólico; levotiroxina; hidróxido de } \\
\text { alumínio; hidróxido de magnésio** }\end{array}$ \\
\hline B & 17 & $\begin{array}{l}\text { butilbrometo de escopolamina + dipirona; dimenidrinato + b6; ranitidina; paracetamol; paracetamol + butilbrometo } \\
\text { de escopolamina; enoxaparina; heparina; pindolol; vitamina d3; cafeina; diclofenaco; penicilina; metronidazol; } \\
\text { cefalexina; loratadina; sulfato de salbutamol; insulina }\end{array}$ \\
\hline C & 15 & $\begin{array}{l}\text { bromoprida; metoclopramida; simeticona; sertralina; fluoxetina; paroxetina; passiflora incarnata + crataegus } \\
\text { oxyacantha + salix alba; risperidona; cafeína + carisoprodol + diclofenaco sódico + paracetamol; metildopa; } \\
\text { budesonida; espiramicina; nitrofurantoína; ômega 3; cálcio }\end{array}$ \\
\hline D & 12 & $\begin{array}{l}\text { dipirona; orfendadrina + dipirona + cafeína; paracetamol + cloridrato de pseudoefedrina; clonazepam; fenobarbital; } \\
\text { bupropiona; fenitoína; fumarato de formoterol diidratado + budesonida; atenolol; prednisona; progesterona; } \\
\text { fluconazol }\end{array}$ \\
\hline$x$ & 2 & etinilestradiol + gestodeno; sulfametoxazol + trimetropina \\
\hline SC & 1 & polivitamínicos*** \\
\hline
\end{tabular}

$\mathrm{SC}=$ sem classificação; *Considerado a classe de maior risco na associação e/ou interação de princípios ativos e/ou possíveis potencializações dos riscos; ${ }^{* *}$ A classificação dos hidróxidos pode variar de A a C, dependendo da concentração e condições da gestante; ${ }^{* * *}$ Em condições específicas e dependendo da formulação, pode pertencer a classe de risco $A$ 
Tabela 4. Uso de outros agentes terapêuticos (chás, agentes terapêuticos tópicos) entre 104 gestantes de Alfenas, MG.

\begin{tabular}{|c|c|c|c|}
\hline Outros agentes terapêuticos & Gestantes $(n=104)$ & $\%$ & Classe(s) de risco(s) FDA \\
\hline \multicolumn{4}{|c|}{ Agentes anti-infecciosos/anti-inflamatórios } \\
\hline Nistatina e miconazol creme* & 1 & 0,97 & $B+C$ \\
\hline Metronidazol** e Nistatina pomada* & 1 & 0,97 & $B+B$ \\
\hline Nistatina pomada & 1 & 0,97 & B \\
\hline Metronidazol geleia* & 1 & 0,97 & B \\
\hline Dexametasona creme & 1 & 0,97 & C \\
\hline Pomada Aciclovir & 1 & 0,97 & C \\
\hline $\begin{array}{l}\text { Agentes não especificados para tratar ITU/corrimen- } \\
\text { tos, hemorroidas, etc.. }\end{array}$ & 12 & 11,5 & - \\
\hline Chás & 9 & 8,65 & $\underline{-}$ \\
\hline Sem relato de uso & 72 & 69,2 & 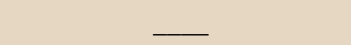 \\
\hline
\end{tabular}

ITU= infecções no trato urinário; *as absorções dos princípios ativos contidos nestas formulações podem ser menores a partir da aplicação tópica; ${ }^{* *}$ Contra indicado no primeiro trimestre para tratar tricomoníase segundo informação do fabricante

Quanto às categorias de risco estabelecidas pelo FDA, a classe de risco mais utilizada entre as 104 entrevistadas foi a $\mathrm{A}(n=75,72,1 \%$ das entrevistadas,), seguida pela $\mathrm{B}(n=64,61,6$ das entrevistadas). Dos 53 diferentes medicamentos utilizados entre os 316 encontrados, as categorias de risco que apresentaram maior diversidade de medicamentos foram B (17 diferentes especialidades), C (15 especialidades) e D (12 especialidades), seguidas de A (6 especialidades) e X (2 especialidades). Por outro lado, neste levantamento foi observado que a frequência de uso dos medicamentos pertencentes ao grupo A (115 medicamentos) foi aproximadamente 3 vezes maior se comparado à frequência de uso do grupo $\mathrm{C}$ (36 medicamentos). Ao se comparar o grupo A com o grupo D (29 medicamentos), os medicamentos do grupo A foram aproximadamente 4 vezes mais utilizados pelas gestantes. Resultados semelhantes foram verificados para os medicamentos do grupo $\mathrm{B}$ se comparados aos do grupo $\mathrm{C}$ e $\mathrm{D}$.

Mengue e cols. (2001) evidenciaram que entre os 9.556 medicamentos utilizados por gestantes, 3.243 (34,0\%) foram incluídos na categoria A, $1.923(22,6 \%)$ na categoria $\mathrm{B}, 3.798(39,7 \%)$ na categoria $\mathrm{C}, 289(3,0 \%)$ na categoria $\mathrm{D}$ e $55(0,6 \%)$ na categoria $\mathrm{X}(16)$. Ainda, Geib e cols. (2007). encontraram que o uso das classes de risco foram: A (53,4\%), B (18,1\%), C (24,46\%), D $(1,47 \%)$ e X $(0,06 \%)(17)$. Guerra e cols. (2008) reportaram um consumo, entre 610 gestantes, de $42,7 \%$ de medicamentos pertencendo à categoria $\mathrm{A} ; 27,1 \%$ à categoria $\mathrm{B}, 29,3 \%$ à categoria $\mathrm{C} ; 0,3 \%$ à categoria $\mathrm{D}$ e nenhum à categoria $\mathrm{X}(14)$. Os dados apresentados por Guerra e cols. (2008) (14), comparados aos desse estudo, e Mengue e cols. (2001) (16) e Geib e cols. (2007) (17), mostram uma notória discrepância apenas quanto à clas- se $\mathrm{C}$, em que aqui é reportada uma frequência da classe de $11,4 \%$ e $28,9 \%$ das gestantes consumiram tal classe.

Quanto às classes terapêuticas relatadas nesse estudo, que mais apareceram nas diferentes classes de risco do FDA, os ansiolíticos, antidepressivos, agentes anti-infecciosos de uso sistêmico e analgésicos foram as mais frequentes (representantes nas classes de risco de A a D), seguidas dos medicamentos com ação sobre o sistema digestivo, anti-hipertensivos, de ação endócrina e vitaminas/suplementos minerais/preparações anti-anêmicas. Complementarmente, nas Tabelas 5 e 6, e no Quadro 3, pode ser observado que, numericamente, as classes mais utilizadas foram: vitaminas/suplementos minerais/preparações anti-anêmicas $(\mathrm{n}=112,35,33 \%)$, analgésicos $(\mathrm{n}=46,14,6 \%)$ e agentes anti-infecciosos de uso sistêmico $(\mathrm{n}=32,10,1 \%)$, consumidas por $72,97 \%, 44 \%$ e $19,2 \%$ das gestantes, respectivamente.

Ainda, dos 316 medicamentos, $30,1 \%$ foram relatados quanto a suas ações sistêmicas, $14,86 \%$ com efeitos sobre o sistema digestivo e $4,46 \%$ sobre o sistema nervoso. Similarmente, Mengue e cols. (2001) encontraram que os medicamentos mais utilizados entre as gestantes entrevistadas foram vitaminas associadas à anti-anêmi$\cos (33,5 \%)$, os medicamentos que atuam sobre o aparelho digestivo $(31,3 \%)$, os analgésicos/anti-inflamatórios $(22,2 \%)$, os anti-anêmicos $(19,8 \%)$ e os antimicrobianos $(11,1 \%)$ (16). Guerra e cols. (2008). mostraram que as classes mais utilizadas entre gestantes foram os anti-anêmicos $(35,6 \%)$, analgésicos $(24,9 \%)$, medicamentos para distúrbios gastrintestinais $(9,1 \%)$ e vitaminas $(7 \%)$ (14). O uso de vitaminas/suplementos minerais/preparações anti-anêmicas pode fazer parte de protocolos visando à promoção de saúde durante a gestação e/ou o 
tratamento de anemias e/ou deficiência de vitaminas e minerais, portanto, justificando os valores aqui apresentados, e os dos estudos supracitados.

Quando considerados os medicamentos representantes das classes terapêuticas, os mais frequentes entre os 316 , como encontrados neste levantamento, foram ácido fólico $(17,72 \%)$, sulfato ferroso $(15,82 \%)$ e paracetamol (12,97\%), como observado na Tabela 5, que também apresenta os respectivos IC(95\%). Em geral, os analgésicos representam uma das classes mais utilizada pela população brasileira. Resultados semelhantes foram também observados por outros pesquisadores (14), em que os princípios ativos usados com maior frequência por gestantes foram: sulfato ferroso $(22,7 \%$ dos medicamentos), paracetamol (13,1\%), ácido fólico (12,9\%); e sulfato ferroso ( $45 \%$ das entrevistadas), paracetamol $(43,4 \%)$ e ácido fólico (13,6\%) (6), indicando que estes três medicamentos são comumente relatados como os mais utilizados durante a gestação.

Tendo em conta as classes de alto risco teratogênico, 24\% $(n=25)$ das mulheres utilizavam medicações constantes classes D ou X, 22,1\% $(n=23)$ utilizavam a $\mathrm{D}$ e $1,9 \%(n=2)$ utilizaram medicamentos da classe $\mathrm{X}$ (estas também utilizaram concomitantemente medicamentos da classe D, Tabela 2).

Em um dos casos em que medicamentos de classe $\mathrm{X}$ foram utilizados (Tabela 2), isso ocorreu por conta de uma situação comumente descrita na literatura médica, também encontrada por Fontoura (2009), em que uma mulher continuou utilizando o método anticoncepcional (representante da classe X, como visto na Tabela 3) por algumas semanas em que ainda não se sabia que estava grávida (19). No outro caso de uso da classe X encontrado neste estudo, um antimicrobiano, o sulfametoxazol + trimetropina, foi utilizado por uma gestante durante o terceiro trimestre de gestação, um período relativamente mais seguro que os dois primeiros trimestres. Contudo, até mesmo neste período, este medicamento deve ser evitado durante a gestação, mas em alguns casos são pesados o possível benefício e também a ausência de alternativas terapêuticas mais seguras para o tratamento, justificando a escolha.

Todos os medicamentos das classes $\mathrm{D}$ e $\mathrm{X}$ foram prescritos, e os valores aqui encontrados para as classes D e X também estão de acordo com aqueles encontrados na literatura $(14,16,17)$.

Em relação ao uso de suplementos, $72 \%$ das entrevistadas utilizavam algum tipo, sendo que uma gestante utilizava dois tipos diferentes. No geral, as vitaminas são classificadas pela FDA como risco A, mas seu uso em superdosagens pode ser prejudicial e estas então passam a ser classificadas como risco $\mathrm{C}$, exceto a vitamina A que passa a ser risco $\mathrm{X}$, ou se consumida concomitantemente com a vitamina $\mathrm{D}$ passa a ser risco $\mathrm{B}$.

Tabela 5. Classes terapêuticas utilizadas entre 104 gestantes de Alfenas, MG

\begin{tabular}{|c|c|c|c|}
\hline Classificação terapêutica, Sistema ATC & $\begin{array}{c}\text { Número de } \\
\text { medicamentos }\end{array}$ & $\begin{array}{c}\% \\
(n=316)\end{array}$ & $\begin{array}{c}\% \text { gestantes sob uso } \\
(n=104)\end{array}$ \\
\hline \multicolumn{4}{|l|}{ Ação sobre o sistema digestivo $(14,86 \%)$} \\
\hline $\begin{array}{l}\text { Anti-ácidos (A02A); Agentes anti-espasmódicos (A03); } \\
\text { Anti-eméticos (A04); Anti-eméticos e antinauseantes } \\
\text { (A04); Medicamentos para distúrbios gastrintestinais } \\
\text { funcionais (A03A); Fármacos para o tratamento da úlcera } \\
\text { péptica (A02B) }\end{array}$ & 47 & 14,86 & 45,31 \\
\hline \multicolumn{4}{|l|}{ Ação sobre o sistema nervoso $(4,46 \%)$} \\
\hline $\begin{array}{l}\text { Agentes anticolinérgicos (N04A); Anti-espasmódicos } \\
\text { associados com analgésicos (A03D); Ansiolíticos (N05B); } \\
\text { Antidepressivos (N06A); Hipnóticos e sedativos (N05C); } \\
\text { Anti-epiléticos (N03); Antipsicóticos (N05A); Relaxantes } \\
\text { musculares de ação periférica (M03A) }\end{array}$ & 14 & 4,46 & 12,56 \\
\hline \multicolumn{4}{|l|}{ Ação sistêmica $(30,1 \%)$} \\
\hline $\begin{array}{l}\text { Agentes antitrombóticos (B01); Anti-hipertensivos } \\
\text { (CO2); Anti-histamínicos de uso sistêmico (R06); Anti- } \\
\text { asmáticos - fármacos para doenças obstrutivas das vias } \\
\text { aéreas (R03); Fármacos utilizados em diabetes (A10); } \\
\text { Outros analgésicos e antipiréticos (N02B); Agentes anti- } \\
\text { inflamatórios e antirreumáticos não-esteróides (M01A); } \\
\text { Corticoides de uso sistêmico (H02); Agentes anti- } \\
\text { infecciosos de uso sistêmico (J) }\end{array}$ & 95 & 30,1 & 78,51 \\
\hline
\end{tabular}




\begin{tabular}{|c|c|c|c|}
\hline Classificação terapêutica, Sistema ATC & $\begin{array}{c}\text { Número de } \\
\text { medicamentos }\end{array}$ & $\begin{array}{c}\% \\
(n=316)\end{array}$ & $\begin{array}{l}\% \text { gestantes sob uso } \\
(n=104)\end{array}$ \\
\hline \multicolumn{4}{|l|}{ Ação endócrina $(2,8 \%)$} \\
\hline $\begin{array}{l}\text { Preparações hormonais sistêmicas, excluindo hormônios } \\
\text { sexuais (H); Hormônios sexuais e moduladores do sistema } \\
\text { genital (G03) }\end{array}$ & 9 & 2,8 & 8,6 \\
\hline \multicolumn{4}{|l|}{ Geral $(35,33 \%)$} \\
\hline $\begin{array}{l}\text { Vitaminas (A11); Suplementos minerais (A12); } \\
\text { Preparações anti-anêmicas (B03) }\end{array}$ & 112 & 35.33 & 72.97 \\
\hline Outros/Não classificáveis $(12,3 \%)$ & 39 & 12,3 & 37,5 \\
\hline Total $(100 \%)$ & 316 & 100 & ------- \\
\hline
\end{tabular}

Tabela 6. Fármacos mais prescritos entre 104 gestantes entrevistadas em Alfenas, MG, Brasil

\begin{tabular}{|c|c|c|c|}
\hline Fármaco (Classificação terapêutica - Sistema ATC) & $\%(n=316)$ & $\mathrm{IC}(95 \%)$ & $\begin{array}{c}\% \text { gestantes } \\
\text { sob uso } \\
(n=104)\end{array}$ \\
\hline Ácido fólico (Preparações anti-anêmicas - B03B) & 17,72 & $13,67-22,39$ & 53,8 \\
\hline Sulfato ferroso (Preparações anti-anêmicas - B03AA07) & 15,82 & $11,98-20,32$ & 48,1 \\
\hline Paracetamol (Analgésicos - N02BE01) & 12,97 & $9,47-17,19$ & 43,3 \\
\hline Butilbrometo de escopolamina (Anti-espasmódicos - A03DB04) & 6 & $0-13,72$ & 18,3 \\
\hline Dimenidrinato + B6 (Anti-eméticos e antinauseante - A04AD) & 6 & $0-13,72$ & 18,3 \\
\hline Cefalexina (Agentes anti-infecciosos de uso sistêmico - J01DB01) & 3,5 & $0-11,22$ & 10,6 \\
\hline Polivitamínico e Poliminerais** & 2,8 & $0-10,52$ & 8,6 \\
\hline Polivitamínico e Poliminerais*** & 2,8 & $0-10,52$ & 8,6 \\
\hline Amoxicilina (Agentes anti-infecciosos de uso sistêmico - J01CA04) & 1,9 & $0-9,62$ & 5,8 \\
\hline Polivitamínico e Poliminerais ${ }^{* * *}$ & 1,9 & $0-9,62$ & 5,8 \\
\hline Progesterona (Hormônios sexuais e moduladores do sistema genital/Progestogênios - G03AC06) & 1,6 & $0-9,32$ & 4,8 \\
\hline Levotiroxina (Terapia para tireoide/Hormônios tiroidianos - H03AA01) & 1,6 & $0-9,32$ & 4,8 \\
\hline Metildopa (Anti-hipertensivos/Agentes anti-adrenérgicos de ação central - C02AB01) & 1,3 & $0-9,02$ & 3,8 \\
\hline Nitrofurantoína (Agentes anti-infecciosos de uso sistêmico - J01XE01) & 1,3 & $0-9,02$ & 3,8 \\
\hline Insulina (Fármacos utilizados em diabetes/Insulinas e análogos - A10A) & 1,3 & $0-9,02$ & 3,8 \\
\hline Bromoprida (Anti-espasmódicos/Propulsivos - A03FA04) & 1,3 & $0-9,02$ & 3,8 \\
\hline Vitaminas/minerais/antianêmicos: & ** Marca A; & *** Marca B; & $\star * * *$ Marca C \\
\hline
\end{tabular}

Tabela 7. Relação entre a idade gestacional (trimestre) e a utilização de medicamentos entre 104 gestantes entrevistadas, em Alfenas, MG, Brasil.

\begin{tabular}{|c|c|c|}
\hline Idade Gestacional & Gestantes entrevistadas $(\boldsymbol{n} / \%)$ & $\begin{array}{c}\text { Gestantes consumindo medicamentos } \\
(\boldsymbol{n} / \%)\end{array}$ \\
\hline & Trimestre (Semanas) \\
\hline $17 / 16,4$ & $30 / 28,8$ & $57 / 54,8$ \\
\hline $104 / 100$ & Primeiro (3-13) & $17 / 16,4$ \\
\hline Segundo & $(14-27)$ & $28 / 27$ \\
\hline Terceiro & $(28-41)$ & $53 / 51$ \\
\hline Total & $104 / 100$ & $98 / 94,2$ \\
\hline
\end{tabular}


Tabela 8. Relação entre a idade gestacional (trimestre) e o uso de medicamentos (classificados pelo FDA*) entre 104 gestantes de Alfenas, MG, Brasil.

\begin{tabular}{|c|c|c|c|c|c|c|}
\hline \multirow{2}{*}{$\begin{array}{c}\text { Gestação } \\
\text { (trimestre) }\end{array}$} & \multicolumn{4}{|c|}{ Classificação FDA) } & \multirow[t]{2}{*}{$\begin{array}{c}\text { Total de } \\
\text { medicamentos (n) }\end{array}$} & \multirow[t]{2}{*}{$\begin{array}{c}\text { Total de } \\
\text { medicamentos }(\%)\end{array}$} \\
\hline & A & B & C & D & & \\
\hline Primeiro & 18 & 23 & 2 & 6 & 49 & 16,3 \\
\hline Segundo & 25 & 26 & 11 & 9 & 71 & 23,6 \\
\hline Terceiro & 72 & 72 & 23 & 14 & 181 & 60,1 \\
\hline Total (n/\%) & 115 & 121 & 36 & 29 & 301 & 100 \\
\hline
\end{tabular}

*com exceção da classe X (2 medicamentos), associações de princípios ativos (2) e não classificáveis (11).

A Tabela 6 e o Quadro 3 mostram todas as vitaminas e minerais encontrados nas bulas dos diversos polivitamínicos consumidos pelas gestantes e suas respectivas classificações pela FDA.

Diferentes estudos têm destacado a importância do uso da suplementação vitamínica e mineral durante a gestação, assim, por exemplo, o ácido fólico é usado como parte de protocolos ou recomendações para o período gestacional (20-22). Lunet e cols. (2008) relataram que entre 836 gestantes portuguesas, o uso destes compostos foi da magnitude de 55,4\% para o ácido fólico, $81,9 \%$ para o ferro e $76,2 \%$ para multivitaminas (20). Em Braço do Norte, SC, Brasil, das 212 gestantes, entrevistadas entre abril de 2012 e junho de 2013, Lunardi-Maia e cols. (2014) reportaram o uso de ácido fólico e sulfato ferroso entre gestantes, observando que $32,5 \%$ não seguiam o protocolo de uso para o ácido fólico e sulfato ferroso e $67,9 \%$ tiveram exposição inadequada aos fármacos (21). Quanto à suplementação com sulfato ferroso, Cesar e cols. (2013) mostraram que das 2557 entrevistadas em Rio Grande, Brasil, durante o ano de $2007,59 \%$ fizeram uso deste suplemento durante a gestação (22).

Quanto à idade gestacional, 16,4\% $(n=17)$ das 104 gestantes estavam, no momento da entrevista, no primeiro trimestre de gestação, $28,8 \%(n=30)$ no segundo trimestre e $54,8 \%(n=57)$ no terceiro trimestre. Todas as 17 entrevistadas que estavam no primeiro trimestre de gestação usavam algum medicamento constante nas classes de risco do FDA (A à D), e apenas 2 gestantes no segundo trimestre e 4 no terceiro trimestre não usaram (Tabela 7).

Com relação à quantidade de medicamentos usados pelas 104 gestantes e, considerado apenas medicamentos constantes nas classes de $\mathrm{A}$ à $\mathrm{D}$, de acordo com a Tabela $8,16,3 \%(n=49)$ dos 301 medicamentos reportados foram usados durante o primeiro trimestre, $23,6 \%$ $(n=71)$ no segundo e, no terceiro trimestre, ocorreu a maior frequência de uso $(60,1 \%, n=181)$, evidenciando uma tendência de uso neste trimestre, que é justificada, ao menos em parte, pelas maiores necessidades terapêuticas deste período e/ou devido à conduta adotada por parte de especialistas, visando evitar o uso de medicamentos no primeiro trimestre da gestação, desde que, algumas alterações, principalmente má formação congênita, possuem maiores chances de ocorrerem neste período (13).

Também neste contexto, um menor consumo de medicamentos das classes $\mathrm{C}$ e $\mathrm{D}$ (que apresentam maiores potenciais de causar teratogênese) foram observados no primeiro trimestre, relativo ao consumo das classes A e B (as percentagens detalhadas para cada classe de acordo com o trimestre são apresentadas na Tabela 8). Confirmando estes achados, foi verificado que houve associação significativa $(p=0,0111)$ entre a idade gestacional e uso de medicação (Tabela 1).

Nesse estudo, com relação aos hábitos de fumar e de ingerir bebidas alcoólicas (Tabela 1), foi observado que $22 \%(n=23)$ das gestantes fumavam e que $20 \%(n$ $=21)$ consumiam algum tipo de bebida alcoólica antes da gravidez; e 11 gestantes afirmaram fumar e consumir bebida alcoólica concomitantemente, antes da gestação. Duas gestantes possuíam histórico de uso de drogas ilícitas, mas afirmaram não fazer uso durante a gestação. Das gestantes que fumavam antes da gestação, 13 continuaram a fumar durante a gestação. Das gestantes que possuíam o hábito de consumir bebidas alcoólicas, 4 continuaram consumindo durante a gravidez. Em nosso estudo, o consumo de álcool ou histórico de tabagismo entre as gestantes não teve correlação com o consumo de medicamentos.

O tabagismo e/ou alcoolismo durante a gestação têm sido apontados como consideráveis causas de complicações maternas e para o feto, incluindo um elevado 
risco de aborto e teratogênese. A ansiedade e depressão estão entre os fatores relatados como associados aos hábitos de fumar e ingerir bebidas alcoólicas, sendo também observado um maior número de medicamentos, usados por gestantes, para controlar estas condições clínicas (23). Neste estudo, as classes dos ansiolíticos e antidepressivos que foram usados pelas gestantes e suas respectivas classes de riscos são apresentadas na Tabela 3, onde é visto uma ampla representatividade destas classes terapêuticas e tendo seus medicamentos enquadrados nas categorias de risco C e D. Em nosso estudo, os medicamentos destas classes terapêuticas foram utilizados por aproximadamente $10 \%$ das 104 gestantes entrevistadas.

No estudo de Bertani e cols. (2015), entre 61 gestantes da cidade de Botucatu, SP, Brasil, entrevistadas, uma frequência de 40,9\% era fumantes, 18 fumantes passivas, 24 gestantes foram classificadas como prováveis/possíveis $(39,3 \%)$ ansiosas e 13 prováveis/possíveis depressivas $(21,3 \%)$, e $21(42,9 \%)$ consumiram álcool durante a gestação (23). Os autores também relacionaram o tabagismo entre as gestantes ao fato das entrevistadas possuírem poucos conhecimentos sobre as consequências do uso de tabaco, sendo que apenas 13 gestantes $(29,6 \%)$ conheciam as consequências pulmonares do tabagismo; somente $2(4,9 \%)$ conheciam os riscos cardiovasculares; e $23(46,9 \%)$ acreditavam que fumar não causa nenhum problema para o feto ou o recém-nascido. No estudo de Geib e cols. (2007), tabagismo no pré-natal foi admitido por $20 \%$ das entrevistadas e consumo de álcool por 17\% (17).

Silva e cols. (2011) observaram em um estudo entre 2007 e 2008, realizado na cidade de Pelotas, RS, Brasil, que $2,1 \%$ de gestantes cometeram abuso com álcool durante a gravidez e, que, entre estas gestantes, $26,3 \%$ deram à luz a crianças com baixo peso, indicando que o abuso de álcool apresenta correlação positiva com baixo peso ao nascer $(p<0.038)$ (24). Ainda, o baixo peso ao nascer não apresentou relação causal com as variáveis socioeconômicas (renda familiar), idade materna, assistência pré-natal, gênero e hábito de fumar $(p>0,05)$, mas sim com abuso de álcool ou prematuridade $(p<0,05)$. Kassada e cols (2013)., mostraram que em 2012, na cidade de Maringá, PR, Brasil, entre 394 grávidas entrevistadas, $18,28 \%$ usavam algum tipo de droga, sendo que 6,09\% usavam álcool, 9,14\% cigarros, 0,51\% Crack, 0,51\% maconha, $1,52 \%$ álcool e cigarros e $0,51 \%$ álcool, cocaína e maconha (25). Em outros países, valores de aproximadamente $18 \%$ para alcoolismo entre gestantes têm sido reportados (26).

A partir dos achados do presente levantamento, são aconselháveis novos estudos para utilização de fármacos mais seguros durante a gravidez, além do uso racional destes, uma vez que a gestante está frequentemente sujeita a complicações que necessitam de intervenção medicamentosa, sendo que para isso, é também necessário haver interação entre as diversas áreas da saúde (interdisciplinaridade) e maiores investimentos no setor.

Como limitações deste estudo e tendências relacionadas ao tema, pode ser destacada a dificuldade para uma unânime e abrangente classificação dos medicamentos, bem como a interpretação dos potenciais riscos. Como observado aqui, uma das especialidades farmacêuticas (polivitamínicos) não se enquadrou em qualquer das classes do FDA. Neste contexto, segundo Law e cols. (2010), as categorias de risco para fármacos usados durante a gestação devem ser interpretadas com cautela, tanto as classificadas pelo FDA quanto pelo Compendium of Pharmaceuticals and Specialties, desde que as informações constantes nelas são algumas vezes insuficientes (18). Assim, tem sido sugerida a adoção de interpretações adicionais e mais específicas, quanto ao uso ou não do medicamento, pesados os potenciais riscos de sua segurança, tais como as constantes na "Motherisk approach", uma abordagem que leva em conta uma profunda avaliação na literatura bem como baseada na experiência clínica e histórico médico das gestantes (18). Outra limitação destacada é o viés de memória das entrevistadas, que pode influenciar nas frequências aqui apontadas. 
Quadro 3. Classificação de risco, segundo a FDA, de vitaminas/minerais encontrados nos polivitamínicos utilizados entre as 104 gestantes entrevistadas

Classe FDA

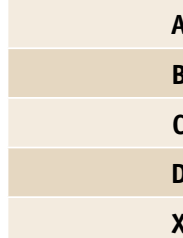

Sem classificação

\section{Vitaminas/Minerais encontrados nos suplementos Polivitamínicos}

Ferro, Vitaminas B2, B3, B6, B12, A, C, Ácido fólico, Ácido pantotênico, Biotina, Cálcio

VitaminaD

Vitamina B1, Vitamina E

Beta caroteno

Nenhum

Nicotinamida, Cromo, Cobre, Iodo, Magnésio, Manganês, Molibdênio, Selênio

\section{CONCLUSÃO}

Um grande número de medicamentos constantes nas 5 classes de risco do FDA foi utilizado durante a gestação, todos prescritos. Além disso, uma considerada frequência de uso de medicamentos das classes de alto risco teratogênico (D e X) foi observada (23\%), com consequências individuais podendo ser sérias e irreversíveis. Além disso, uma grande proporção de gestantes utilizou mais de um medicamento. Ainda, o uso dos polivitamínicos, frequente entre as entrevistadas, mesmo fazendo parte de protocolos ou recomendações para o período gestacional, deve ser cuidadoso e em doses baixas, uma vez que doses excessivas podem ser prejudiciais ao feto. Não houve associação estatística entre o uso de medicamentos de qualquer das classes e variá- veis socioeconômicas ou tabagismo/consumo de álcool. Contudo, foi observado um elevado número de gestantes com hábitos de tabagismo e de consumo de bebidas alcoólicas, inclusive com preocupantes proporções de entrevistadas que mantiveram estes hábitos durante o período gestacional. Houve relação de dependência entre o uso de medicamentos e idade gestacional, tendo sido observado um maior consumo no terceiro trimestre de gestação. Apesar disto, houve também um considerável consumo de medicamentos no primeiro trimestre, período considerado crítico quanto ao possível efeito teratogênico dos fármacos, principalmente das classes D e X. Portanto, o uso de medicamentos de risco por gestantes é uma realidade, mesmo por aquelas que realizam atendimento médico durante o período pré-natal, visto que todos os medicamentos relatados nesse estudo foram prescritos.

\section{REFERÊNCIAS}

1. Gualda DMR. O significado dos indicadores de qualidade: uma abordagem perinatal. São Paulo: Icone; 1998.

2. Rezende J. Montenegro CAB. Obstetrícia. $13^{\mathrm{a}}$ ed. Rio de Janeiro: Guanabara Koogan; 2016.

3. Piccinini CA, Lopes RS, Gomes AG, De Nardi T. Gestação e a constituição da maternidade. Psicol Estud. 2008;13(1): 63-72.

4. Hall JE, Guyton AC. Tratado de Fisiologia Médica. 13 Ed. Elsevier; 2017.

5. Nesbitt Jr REL, Aubry RH. High risk obstetrics. II. Value of semiobjetive grading system in identifying the vulnerable group. Amer J Obstet Gynecol. 1969;103: 972-85.

6. Melo SCCS, Pelloso SM, Carvalho MDB, Oliveira NLB. Uso de medicamentos por gestantes usuárias do Sistema Único de Saúde. Acta Paul Enferm. 2009;22 (1): 66-70. DOI: $10.1590 / \mathrm{S} 0103-21002009000100011$
7. Nascimento ER, Paiva MS, Rodrigues QP. Avaliação da cobertura e indicadores do Programa de Humanização do Pré-natal e Nascimento no município de Salvador, Bahia, Brasil. Rev Bras Saúde Materno Infantil. 2007;7(2):191197. DOI: $10.1590 / \mathrm{S} 1519-38292007000200010$

8. Demitto MO, Gravena AAF, Dell'Agnolo CM, Antunes MB, Pelloso SM. Gestação de alto risco e fatores associados ao óbito neonatal. Rev Esc Enferm USP. 2017;51:e03208. DOI: 10.1590/S1980220X201601470320

9. Nunes JT, Gomes KRO, Rodrigues MTP, Mascarenhas MDM. Qualidade da assistência pré-natal no Brasil: revisão de artigos publicados de 2005 a 2015 . Cad Saúde Colet. 2016;24(2):252-261. DOI: 10.1590/1414462X201600020171

10. Costa AM, Guilherme D, Walter MIMT. Atendimento a gestantes no Sistema Único de Saúde. Rev. Saú- 
de Pública. 2005;39 (5):1-7.DOI: 10.1590/S003489102005000500011

11. BRASIL. Ministério da Saúde. Secretaria de Atenção à Saúde. Departamento de Ações Programáticas Estratégicas. Gestação de alto risco: manual técnico. $5^{\text {a }}$. ed. Brasília: Editora do Ministério da Saúde; 2010.

12. Fefer E. Uso racional de medicamentos. In: Bermudez JAZ, Bonfim JRA. Medicamentos e a reforma do setor saúde. São Paulo: Hucitec/Sobravime, p. 45-55; 1999.

13. Baldon JP, Correr CJ, Melchiors AC, Rossignoli P, Fernandez-Llimos F, Pontarolo R. Conhecimento e atitudes de farmacêuticos comunitários na dispensação de medicamentos para gestantes. Pharmacy Practice. 2006; 4 (1): 38-43.

14. Guerra GCB, Da Silva AQB, França LB, Assunção PMC, Cabral RX, Ferreira AAA. Utilização de medicamentos durante a gravidez na cidade de Natal, Rio Grande do Norte, Brasil. Rev Bras Ginecol Obstet. 2008; 30 (1): 128. DOI: $10.1590 / \mathrm{S} 0100-72032008000100003$

15. Osorio-de-Castro CGS, Paumgartten FJR, Silver LD. O uso de medicamentos na gravidez. Ciênc Saúde Coletiva. 2004; 9 (4): 987-996. DOI: 10.1590/S141381232004000400019

16. Mengue SS, Schenkel EP, Duncan BB, Schmidt MI. Uso de medicamentos por gestantes em seis cidades brasileiras. Rev Saúde Pública. 2001; 35 (5): 415-20. 10.1590/ S0034-89102001000500002

17. Geib LTC, Vargas-Filho EF, Geib D, Mesquita DI, Nunes ML. Prevalência e determinantes maternos do consumo de medicamentos na gestação por classe de risco em mães de nascidos vivos. Cad Saúde Pública. 2007; 23 (10): 2351-2362. DOI: 10.1590/S0102-311X2007001000010.

18. Law R, Bozzo P, Koren G, Einarson A. FDA pregnancy risk categories and the CPS. Do they help or are they a hindrance? Can Fam Physician. 2010; 56: 239-241.

19. Fontoura A. Utilização de medicamentos por gestantes em atendimento pré-natal em uma maternidade do mu- nicípio de Ribeirão Preto - SP. 121f. Dissertação (mestrado). Faculdade de ciências Farmacêuticas de Ribeirão Preto, 2009.

20. Lunet N, Rodrigues T, Correia S, Barros H. Adequacy of prenatal care as a major determinant of folic acid, iron, and vitamin intake during pregnancy. Cad Saúde Pública. 2008; 24 (5): 1151-1157. DOI: 10.1590/S0102311 X2008000500022

21. Lunardi-Maia T, Schuelter-Trevisol F, Galato D. Uso de medicamentos no primeiro trimestre de gravidez: avaliação da segurança dos medicamentos e uso de ácido fólico e sulfato ferroso. Rev Bras Ginecol Obstet. 2014; 36 (12): 541-7. DOI: 10.1590/SO100-720320140005051

22. Cesar JA, Dumith SC, Chrestani MAD, Mendoza-Sassi RA. Iron supplementation among pregnant women: results from a population-based survey study. Rev Bras Epidemiol. 2013; 16 (3): 729-36. DOI: 10.1590/S1415$790 \times 2013000300016$

23. Bertani AL, Garcia T, Tanni SE, Godoy I. Preventing smoking during pregnancy: the importance of maternal knowledge of the health hazards and of the treatment options available. J Bras Pneumol. 2015; 41 (2): 175-181. DOI: $10.1590 / \mathrm{S} 1806-37132015000004482$

24. Silva ID, Quevedo LdeA, Silva RA, Oliveira SS, Pinheiro RT. Association between alcohol abuse during pregnancy and birth weight. Rev Saude Publica.. 2011; 45 (5): 864-9. DOI: 10.1590/S0034-89102011005000062

25. Kassada DS, Marcon SS, Pagliarini MA, Rossi RM. Prevalence of drug abuse among pregnant women. Acta Paulista Enferm. 2013; 26 (5): 467-71. DOI: 10.1590/ S0103-21002013000500010

26. Onah MN, Field S, Van Heyningen T, Honikman S. Predictors of alcohol and other drug use among pregnant women in a peri-urban South African setting. Int J Ment Health Syst. 2016; 10: 38. DOI: 10.1186/s13033-0160070-x. 